\title{
A Roadmap for Implementation of Patient-Centered Digital Outcome Measures in Parkinson's Disease Obtained Using Mobile Health Technologies
}

\author{
Alberto J. Espay, MD, MSc, ${ }^{1 *}$ (1) Jeffrey M. Hausdorff, PhD, ${ }^{2,3,4}$ Álvaro Sánchez-Ferro, MD, MSc, ${ }^{5}$ (10) \\ Jochen Klucken, MD, ${ }^{6,7}$ Aristide Merola, MD, PhD, ${ }^{1}$ (D) Paolo Bonato, PhD, ${ }^{8}$ Serene S. Paul, PhD, BAppSc(Phty)(Hons), ${ }^{9}$ \\ Fay B. Horak, PhD, PT, ${ }^{10,11}$ Joaquin A. Vizcarra, MD, ${ }^{1}$ Tiago A. Mestre, MD, MSc, ${ }^{12}$ (ID Ralf Reilmann, MD, PhD, ${ }^{13,14,15}$ (iD \\ Alice Nieuwboer, PhD, ${ }^{16}$ E. Ray Dorsey, MD, MBA, ${ }^{17}$ Lynn Rochester, PhD, ${ }^{18,19}$ Bastiaan R. Bloem, MD, PhD, ${ }^{20}$ and \\ Walter Maetzler, $\mathrm{MD}^{21}$ on behalf of the Movement Disorder Society Task Force on Technology \\ ${ }^{1}$ James J. and Joan A. Gardner Family Center for Parkinson's Disease and Movement Disorders, University of Cincinnati, \\ Cincinnati, Ohio, USA \\ ${ }^{2}$ Center for the Study of Movement, Cognition, and Mobility, Department of Neurology, Tel Aviv Sourasky Medical Center, Tel Aviv, Israel \\ ${ }^{3}$ Department of Physical Therapy, Sackler Faculty of Medicine and Sagol School of Neuroscience, Tel Aviv University, Tel Aviv, Israel \\ ${ }^{4}$ Rush Alzheimer's Disease Center and Department of Orthopedic Surgery, Rush University, Chicago, Illinois, USA \\ ${ }^{5}$ HM CINAC, Hospital Universitario HM Puerta del Sur, Móstoles, Madrid, Spain \\ ${ }^{6}$ Department of Molecular Neurology, University Hospital Erlangen, Friedrich-Alexander University Erlangen-Nürnberg, Erlangen, Germany \\ ${ }^{7}$ Fraunhofer Institut for Integrated Circuits, Digital Health Pathway Research Group, Erlangen, Germany \\ ${ }^{8}$ Department of Physical Medicine and Rehabilitation, Harvard Medical School, Boston, Massachusetts, USA \\ ${ }^{9}$ Discipline of Physiotherapy, Faculty of Health Sciences, The University of Sydney, Sydney, New South Wales, Australia \\ ${ }^{10}$ Department of Neurology, Oregon Health \& Science University, Portland Veterans Affairs Medical System, Portland, Oregon, USA \\ ${ }^{11}$ APDM, Inc, Portland, Oregon, USA \\ ${ }^{12}$ Parkinson's Disease and Movement Disorders Center, Division of Neurology, Department of Medicine, The Ottawa Hospital Research \\ Institute, University of Ottawa, Ottawa, ON, Canada \\ ${ }^{13}$ George-Huntington-Institute, Technology Park, Muenster, Germany \\ ${ }^{14}$ Department of Radiology, University of Muenster, Muenster, Germany \\ ${ }^{15}$ Department of Neurodegenerative Diseases and Hertie-Institute for Clinical Brain Research, University of Tuebingen, Tuebingen, Germany \\ ${ }^{16}$ Neuromotor Rehabilitation Research Group, Department of Rehabilitation Sciences, KU Leuven, Leuven, Belgium \\ ${ }^{17}$ Department of Neurology, University of Rochester Medical Center, Rochester, New York, USA \\ ${ }^{18}$ Institute of Neuroscience, Newcastle University, Newcastle Upon Tyne NE4 5PL, UK \\ ${ }^{19}$ Newcastle upon Tyne Hospitals National Health Service Foundation Trust, Newcastle upon Tyne, UK \\ ${ }^{20}$ Radboud University Medical Center, Donders Institute for Brain, Cognition and Behaviour, Department of Neurology, \\ Nijmegen, The Netherlands \\ ${ }^{21}$ Department of Neurology, Christian-Albrechts University, Kiel, Germany
}

\begin{abstract}
Obtaining reliable longitudinal information about everyday functioning from individuals with Parkinson's disease (PD) in natural environments is critical for clinical care and research. Despite advances in mobile health technologies, the implementation of digital outcome measures is hindered by a lack of consensus on the type and scope of measures, the most appropriate approach for data capture (eg, in clinic or at home), and the extraction of timely information that meets the needs of patients, clinicians, caregivers, and health care regulators. The Movement Disorder Society
\end{abstract}

Task Force on Technology proposes the following objectives to facilitate the adoption of mobile health technologies: (1) identification of patient-centered and clinically relevant digital outcomes; (2) selection criteria for device combinations that offer an acceptable benefit-to-burden ratio to patients and that deliver reliable, clinically relevant insights; (3) development of an accessible, scalable, and secure platform for data integration and data analytics; and (4) agreement on a pathway for approval by regulators, adoption into e-health systems and implementation

\footnotetext{
*Correspondence to: Dr. Alberto J. Espay, Gardner Family Center for Parkinson's Disease and Movement Disorders, Department of Neurology, University of Cincinnati, 222 Piedmont Avenue, Cincinnati, $\mathrm{OH}$ 45219, USA; E-mail: aespay@gmail.com.
}

Relevant conflicts of interests/financial disclosures: A workshop for completion of this article took place in Hong Kong, on October 9, 2019, after the Movement Disorder Society Annual Meeting.
Room charges and room accommodations were covered for the participants.

Received: 10 December 2018; Revised: 2 February 2019; Accepted: 28 February 2019

Published online 00 Month 2019 in Wiley Online Library (wileyonlinelibrary.com). DOI: 10.1002/mds.27671 
by health care organizations. We have developed a tentative roadmap that addresses these needs by providing the following deliverables: (1) results and interpretation of an online survey to define patient-relevant endpoints, (2) agreement on the selection criteria for use of device combinations, (3) an example of an open-source platform for integrating mobile health technology output, and (4) recommendations for assessing readiness for deployment of promising devices and algorithms suitable for regulatory approval. This concrete implementation guidance, harmonizing the collaborative endeavor among stakeholders, can improve assessments of individuals with PD, tailor symptomatic therapy, and enhance health care outcomes. (C) 2019 International Parkinson and Movement Disorder Society

Key Words: mobile health technologies; Parkinson's disease; remote monitoring; wearable technology
In 2016, the International Parkinson and Movement Disorders Society Task Force on Technology published a summary of the challenges and opportunities related to the integration of technologies into the clinical management of PD. ${ }^{1}$ Despite the increasing miniaturization and portability of mobile health technologies and despite the worldwide increase in deployment of commercially available devices, ${ }^{2}$ there remains a large gap in their adoption and wide-scale implementation in both care and research. ${ }^{3}$ The mission of this task force is to develop a framework for the development, accessibility, and long-term adherence of mobile health technologies to enhance care and research objectives related to PD. For this purpose, mobile health technologies are defined here as "wearable" or portable devices that can provide objective measures and that include mobile and digital applications as well as body-worn (adhered to a body surface) or frequently used (eg, smartphone) patient-centered devices. This inclusion of mobile and digital interfaces captures the spectrum of devices used by patients irrespective of how they are worn. The final product, as outlined later, is meant to integrate the needs of all stakeholders but be flexible enough to adapt to individual patient needs.

Mobile health technologies have to date integrated only partially into clinical practice and clinical trials, which continue to rely on clinical scales and episodic assessments made in somewhat artificial environments for primary and secondary endpoints. ${ }^{4,5}$ People with PD are particularly prone to performance bias, ${ }^{6}$ as exemplified by the improved performance of their movements when observed in clinical practice. To develop digital outcomes with functional relevance to patients, further proliferation of independent devices that merely target narrow, disconnected aspects of behavior (eg, number of steps) need to be reconsidered in favor of comprehensive longitudinal tracking of patientcentered motor and nonmotor data in home and community settings. Interoperable platforms with communication standards that integrate different devices by providing application programming interfaces ${ }^{7}$ stand to facilitate regulatory approval and adoption by health care organizations.

The purpose of this position paper is to propose a roadmap for the development of patient-centered digital outcomes and their integration into both clinical care ${ }^{8}$ and research that is sensitive to the needs of all relevant stakeholders, most critically patients. The final product aims to facilitate patient self-monitoring and clinicianbased tailoring of symptomatic therapy and to serve as objective endpoints in clinical research, initially as surrogate and exploratory outcomes, but with time perhaps even as primary outcomes. We envision that the roadmap proposed here will likely influence these important areas of research and clinical management. Important related areas that are outside the purview of this roadmap are the use of mobile health technologies for supporting the clinical diagnosis (or aim at serving as a diagnostic test), measuring the underlying neurodegenerative progression (as disentangled from fluctuations in motor or nonmotor behaviors), ${ }^{9}$ detecting prodromal symptoms at a population level, ${ }^{10,11}$ integrating digital outcomes into closed-loop treatment systems to assist in timing and dosage of therapy, or selecting patient subgroups for testing of future disease-modifying treatments.

\section{Current Gaps in the Use of Mobile Health Technologies}

Digital measures derived from wearables/mobile technologies and applications are slowly starting to emerge as secondary or exploratory outcome measures in the context of clinical trials ${ }^{4,12}$ and, to a lesser extent, as treatment targets in clinical care. Most often, digital outcomes have been developed (1) to capture constructs of interest in isolation (eg, tremor or bradykinesia) ${ }^{13}$ without "painting a global picture" and without focusing on patient-centered outcomes; (2) by developers working in isolation from patients, clinicians, or scientific societies; and (3) exclude the wide range of nonmotor features, which are prominent sources of disability for many patients. The adoption of mobile health technologies has been hindered by the presentation and interpretation of the data, often in relation to a population mean rather than to a patient's own baseline and disconnected from patients' functional disability levels. Patient compliance and technology illiteracy have been poorly addressed, particularly when it comes to wearing multiple sensors for long periods of time. An 
exception was a recent study that addressed the long-term compliance of patients to wear a smartwatch, a body-worn sensor, and a smartphone; in this study, a helpdesk to support patients proved a critical strategy to improve adherence. ${ }^{14}$ Another pitfall is the aspirational development of mobile health technologies as fulfilling diagnostic needs. ${ }^{15}$ Although technologies can be harnessed for validating patient-centered outcomes and for supporting a clinical diagnosis, they remain inadequate as stand-alone measures for "diagnostic accuracy." Aiming at fulfilling this goal perpetuates the concept that the many molecular subtypes subsumed within the clinical diagnosis of PD can be unified by an ideal set of behavioral features.

\section{Validation}

A separate challenge exists for the process of validation of mobile health technologies because we would expect them to be more discriminative or sensitive than previously developed clinical scales for motor and nonmotor symptoms. A classic validation paradigm would require the outcomes of mobile technology to "correlate" with these "gold standard" clinical scales, but this should not necessarily be the case. Currently available scales may function well to capture differences at the group level, but may be less suitable to capture changes within an individual. This is where mobile technologies have the potential to excel, perhaps justifying imperfect correlations with clinical scales simply because the objective measurements outperform the more subjective clinical assessments, which are prone to substantial inter- and intrarater variability. In other words, large differences or large detection gaps between digital outcomes and existing scales are in fact desirable because both capture different and perhaps even complementary domains (eg, as the "Mobile Parkinson Disease Score" obtained with smartphones and data analyzed with machine learning). ${ }^{16}$ To be validated, nevertheless, mobile health technologies will require such aspects as accuracy (laboratory validity), reliability (test-retest within and between sensors), sensitivity, and minimal clinically significant difference for any endpoint of interest when tested against direct patient input or any robust measure of clinical meaningfulness (eg, a pull test to compare a new digital biomarker for balance). For clinical studies, the greater precision will allow a greater signal-to-noise ratio for endpoints of interest and a subsequent reduction in the number of patients required for enrolling in clinical trials.

\section{Integration and Standardization}

Currently, to comprehensively capture several motor and nonmotor measures, clinicians need to combine multiple mobile health technologies from different, noncompatible manufacturers, operating on separate platforms. Moreover, the unsupervised, unstructured setting in which wearable-derived measures are obtained introduces confounding variables that cannot be as easily controlled as in the well-structured, more "repeatable" environment of a clinic or research laboratory. These challenges to interpretation and scoring are believed to be outweighed by the ostensible superiority of continuous monitoring and the increasing reliance on big data, according to which sophisticated analytic systems can extract signals of presumed relevance from background noise and as such supplement careful history and neurological examination. A word of caution here is that "big data" do not necessarily equate to "good data" and that spurious and irrelevant conclusions can be reached from big data analytics designed to "uncover hidden patterns.",

\section{Unmet Needs and the Required Levels of Development}

This task force consensus paper addresses 2 major unmet needs regarding the interface between technology and clinical evaluation for care and research. One is the ability to use multiple devices to capture data collected in "free-living" conditions that are relevant to the patient's functioning. The other is the data integration into open-source systems designed to generate individualized feedback to patients, clinicians, researchers, and caregivers. Such developments should be responsive to the needs of all stakeholders caring for PD and should include a pathway for regulatory approval, adequate licensing protection, appropriate reimbursement, and wide patient access using a model that allows for sustainability and growth.

In particular, we address 4 specific needs.

\section{Defining Relevant Patient-Centered Digital Targets and Outcomes to Be Captured With Mobile Health Technologies (What to Measure)}

Behavioral measurements should be relevant to the patient. This patient-centered scope maximizes the likelihood of acquiring data that facilitate clinically important decision-making by the clinician and promote long-term adherence by the patient. Furthermore, it defines a major boundary: not anything that can be measured should be measured. Patient-centered measurement of the presence and severity of motor and nonmotor symptoms should focus on how they impair activities of daily living (ADLs). ${ }^{18}$ Instrumental ADLs can include symptoms ("impairments") and activities ("disabilities"), the latter being typically more reliable for self-reporting measures. ${ }^{19}$ Domains deemed patient-relevant cannot be identified by clinicians or researchers alone. Nonetheless, selected nonmotor endpoints, such as sleep and heart rhythm monitors-both included in most common smartwatches - can be made available for shared decision-making because of their clear 
relevance, even when patients do not prioritize these domains themselves.

\section{Selection Criteria to Guide the Choice of Mobile Health Technology (How to Measure)}

Mobile health technologies should be unobtrusive to patients and capable of capturing the phenomena of interest at intervals that balance patient burden and accuracy. Besides validation issues (this is covered by point 4), a critical component of developing useful mobile health technologies is the standardization of sensor measurements to create an established, broadly accepted common set of metrics. Addressing the critical questions of minimal precision and uniformity of instruments (eg, is $1 \mathrm{~m} / \mathrm{s}$ captured with device A the same as with device B?) as well as the reliance on one versus multiple sensors are vital and could help establish minimal guidelines for adequacy of sensors. In addition, optimal outcomes of algorithms will depend on the adequate balance between prescribed, action-dependent tasks (such as finger tapping, spiral drawing, digital diaries) and natural, action-independent behaviors (ie, passive tracking) and the contextual environment where some measurements occur (specifically, in supervised vs unsupervised settings).

\section{Web-Based, Open-Source, Modular, Scalable, and Secure Platforms for Data Analysis, Integration, and Visualization (What to Display)}

Patient-centered and clinically relevant digital outcomes collected through mobile health technologies would ideally be analyzed to anticipate periods of impairment in ADLs or instrumental ADLs before they occur, helping to individualize both reactive and proactive/preemptive clinical decisions. Such analysis should be integrated and summarized in a display format that is individualized, palatable, and visually intuitive in the context of real-life conditions so that it serves the needs of the end users (ie, patients and the professional team that treats them). Moreover, by not affecting proprietary algorithms from a source device, an open-source/open-access concept could stimulate developmental aspects of sensors, software, algorithms, visualization, and communication tools while promoting licensing as well as protecting intellectual property.

\section{Establish a Roadmap for Regulatory Approval and Adoption Into Health Care Systems (How to Disseminate)}

Demonstration of the utility to providers and patients and efficiencies in data processing will facilitate the integration of mobile health technologies into digital health models with subsequent approval by regulators and adoption by health care delivery organizations. Its integration with digital applications will be critical given the increasing relevance of telemedicine and other types of medicine that use data from the home environment in replacing or supplementing traditional models of patient visits with health professionals.

\section{Proposed Roadmap}

The MDS Task Force on Technology has developed a tentative roadmap that addresses these 4 defined needs by providing the milestones and deliverables. Both the "vision" and "process" for this roadmap were conceptualized by consensus among the task force members, with milestones representing the vision and developmental steps representing the process (Fig. 1).

\section{Relevant Patient-Centered Digital Targets and Outcomes}

To determine patient-defined targets and outcomes, adaptable to individual patient needs, data derived from surveys of more than 10,000 patients through the Michael J Fox Foundation's Fox Insight project have been identified to extract patient-relevant targets. Pioneer work in this cohort used free text responses to the following questions: "What bothers you the most about your PD?" "In what way does this problem bother you by affecting your daily functioning?" The answers were analyzed with natural language processing and machine learning approaches and identified 4 motor (tremor, rigidity, bradykinesia, postural instability) and 6 nonmotor (sleep, fatigue, cognition, mood, pain, constipation) symptoms as patient relevant. ${ }^{20}$ Additional patient cohorts should also be considered to account for geographical and social diversity. ${ }^{21}$ Importantly, the standardization of patient-relevant outcomes poses the risk of losing individualization and will require safeguard mechanisms to ensure that outcomes are tailored to individual patient needs. Some domains may benefit from a clinician's perspective to avoid overlooking important phenomena patients may not recognize when they are just emerging (eg, freezing of gait, eye motility disturbances, postural deficits with deviations of the center of mass, impulsivity, multitasking deficits and reduced attention contributing to postural instability and falls, and nocturnal events/dream enactment behaviors), progressively subtle changes (eg, gradual changes in physical activity), potentially severe events (eg, complex dyskinesias, behavioral fluctuations), and nonmotor manifestations (eg, nocturnal monitoring for sleep-related movements, skin impedance or heart-rate variability for autonomic impairment). Personalization through digital diaries (e-diaries) could serve to expand the range of the latter category. The MDS Task Force on Technology will invite patients, caregivers, and representatives of advocacy organizations to further refine these patient-centered digital targets/outcomes. 


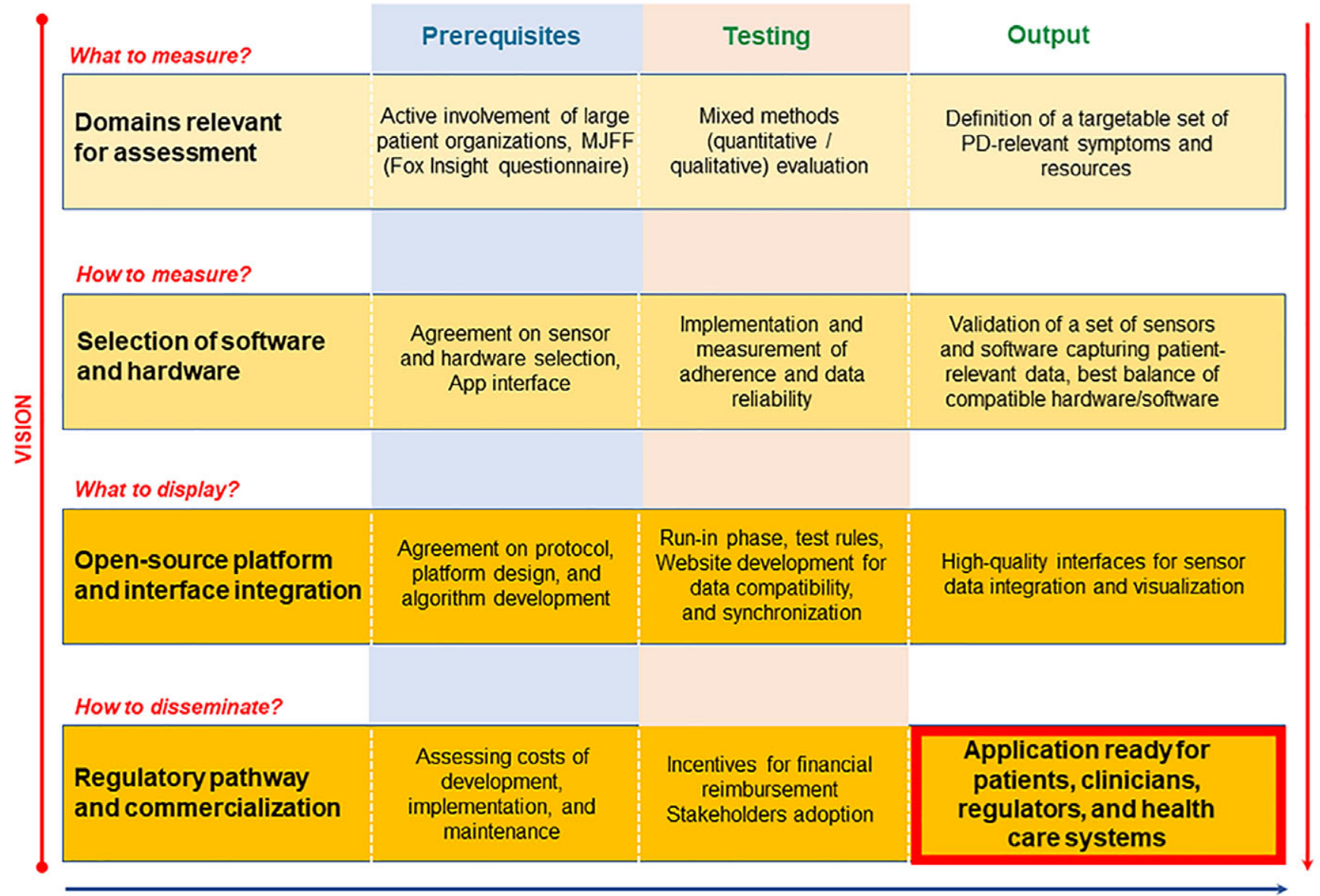

PROCESS

FIG. 1. Levels and phases of development of patient-relevant mobile health technologies. Early to late milestones ("vision") are organized vertically, from top to bottom, and tentative high-level technical steps horizontally, from left to right ("process"). MJFF, The Michael J. Fox Foundation. [Color figure can be viewed at wileyonlinelibrary.com]

\section{Proposal for Selection Criteria for Useful Sensors/Hardware Combinations}

Selection criteria for data collection in real life and real time must address the needs of the user (eg, comfort and user requirements to achieve good compliance), other stakeholders (eg, provide research-grade sensitive and specific outcomes), and technical aspects (eg, battery life, data storage, compatibility with other systems). Also, if the monitoring period is to be over years and/or patients have to wear more than one device, periodic monitoring may be more successful than continuous assessment. Although continuous monitoring appears attractive, systematic evaluation of the benefits and trade-offs of longer (eg, 1 month, 6 months) versus shorter (eg, 1 day, 1 week) durations have not yet been determined. A set of criteria (Table 1) is proposed as the basis for the evaluation of potentially meaningful and relevant devices for use by various stakeholders. Mobile health technology developers will be asked to provide detailed information about these aspects when a system is considered for use by the community and to satisfy platform compliance and assist regulatory needs.

\section{Open-Source Platform Standards for Mobile Health Technologies}

Innovative mobile health technologies have data platforms that transfer measured outcomes or targets to the user, which can be, depending on the application, the patient (eg, via the electronic health record) ${ }^{7,8}$ or caregiver, members of the health care provider team, or insurance or public organizations. Unfortunately, a joint and interoperable platform standard does not exist, neither for technical features nor for adaptability to various clinical or health administration users. In collaboration with method experts from academy and industry, we elaborate on recommendations for a platform standard that could enable a centralized, open-source, web-based structure where mobile health technologies with different technology standards and requirements can be integrated. These recommendations will satisfy the following criteria: (1) userfriendly accessibility; (2) support of scalability and exchangeability; (3) data storage meeting regulatory and security standards; (4) periodic evaluation of calibration, with validation strategies supporting evolving technologies and algorithms; and (5) data access and sharing to include 
ESPAY ET A L

TABLE 1. User-based considerations for choosing data collection methods with mobile health technologies

\begin{tabular}{|c|c|c|c|}
\hline & Patient/caregiver & Health care provider & Researcher \\
\hline Number of sensors & $\begin{array}{l}\text { Minimal number of sensors and } \\
\text { easy-to-access location/s }\end{array}$ & $\begin{array}{l}\text { Number of sensors and locations based } \\
\text { on clinical purpose }\end{array}$ & $\begin{array}{l}\text { Number and location of sensors based on } \\
\text { targeted accuracy }\end{array}$ \\
\hline Sensor burden & $\begin{array}{l}\text { Minimal patient and caregiver burden over } \\
\text { a long time }\end{array}$ & Minimal clinician burden over a long time & $\begin{array}{l}\text { Potentially greater burden in patients and } \\
\text { clinicians over a short time }\end{array}$ \\
\hline Frequency & $\begin{array}{l}\text { Less frequent use to enhance adherence } \\
\text { during data capture }\end{array}$ & Frequency depending on use of data & $\begin{array}{l}\text { More frequent use to ensure, eg, high } \\
\text { signal-to-noise ratio }\end{array}$ \\
\hline Targets & $\begin{array}{l}\text { 1-2 domains at low- frequency intervals, } \\
\text { based on identified problems }\end{array}$ & $\begin{array}{l}\text { Possibly 1-2 domains at periodic intervals, } \\
\text { according to patient's and clinician's } \\
\text { goals }\end{array}$ & $\begin{array}{l}\text { Likely multiple domains at frequent } \\
\text { intervals, according to research } \\
\text { objectives }\end{array}$ \\
\hline User friendliness & $\begin{array}{l}\text { Easy to use, ready (ideally } 24 / 7 \text { ) access to } \\
\text { helpdesk to facilitate compliance and } \\
\text { minimal manual skill level required to } \\
\text { operate the system }\end{array}$ & $\begin{array}{l}\text { Easy to use in clinical practice; helpdesk } \\
\text { to troubleshoot range of potential } \\
\text { problems. Facilitate patient compliance } \\
\text { by reviewing data }\end{array}$ & $\begin{array}{l}\text { Usability and compliance-less of an issue } \\
\text { for fully supervised sessions; will have } \\
\text { to ensure ease of use to facilitate } \\
\text { patient compliance for unsupervised } \\
\text { monitoring }\end{array}$ \\
\hline $\begin{array}{l}\text { Supervised vs } \\
\text { unsupervised }\end{array}$ & $\begin{array}{l}\text { Unsupervised data collection ensured by } \\
\text { friendly, acceptable device to user }\end{array}$ & $\begin{array}{l}\text { Reliance only on unsupervised data } \\
\text { collection }\end{array}$ & $\begin{array}{l}\text { Reliance on supervised and unsupervised } \\
\text { data collection }\end{array}$ \\
\hline $\begin{array}{l}\text { Desirable technical } \\
\text { aspects }\end{array}$ & \multicolumn{2}{|c|}{$\begin{array}{l}\text { Long battery life, low charging, easy or automatic uploading and downloading, small } \\
\text { size, low weight, water-proof. Low level of expertise to use and understand output }\end{array}$} & $\begin{array}{l}\text { Battery life, need for charging, size, and } \\
\text { weight less critical for supervised/short } \\
\text { duration sessions. High level of } \\
\text { expertise to analyze }\end{array}$ \\
\hline Validation & $\begin{array}{l}\text { Must show correlation with global } \\
\text { patient-centered scales for the } \\
\text { appropriate domains }\end{array}$ & $\begin{array}{l}\text { Monitoring of motor fluctuations and } \\
\text { medication titration }\end{array}$ & $\begin{array}{l}\text { May not strongly correlate with the total } \\
\text { or even specific items of such gold } \\
\text { standards as the UPDRS. Observation } \\
\text { or video analysis may be needed }\end{array}$ \\
\hline
\end{tabular}

governance and right management regarding privacy and ownership/licensing of medical data. Such a process for data platform standardization will ensure adequate data management for self-management (of the patient), care and research applications, support regulatory bodies by providing testable quality standards for licensing, and certification and approval procedures of new health care technologies.

The MDS Task Force on Technology in collaboration with the Rating Scales Electronic Development Ad Hoc Committee is developing an early, proof-of-concept "integration" effort through the development of an e-diary/tracker for PD. Such an initiative could be an example for future efforts and serve to answer the following 2 critical questions: (1) Can a central body such as MDS invest the necessary resources for the development, maintenance, and administration (including dynamic decision-making on which elements to include or exclude) of a needed interoperable open-source, securitycompliant platform into which mobile health technologies integrate? (2) Should MDS develop criteria for existing and emerging applications as "clinically meaningful" and "sufficiently accurate" about which sensitivity, specificity, and diagnostic accuracy cannot be ascertained given the problems with using current clinician-rated rating scales as the gold standard?

\section{Regulatory Pathways and Commercialization}

We expect that standards for integrated health care technology platforms will increase the adoption of mobile health technologies into clinical care and clinical trials.
Unifying open-source platforms are expected to encourage commercial developers to further innovate both the hardware and proprietary algorithms because they can read and synchronize data from multiple devices. Furthermore, such platforms will need to be accessible and user friendly by all users to allow clinicians and patients to shop for any combination of devices, from any company, in the hope of finding whichever meets their individual needs. Importantly, they should also be attractive to companies as a "marketplace" in which a range of hardware solutions competes for patients' and clinicians' interests, without concerns for interdevice compatibility or ability to synchronize data from other devices. Future research studies will have to show the cost-effectiveness of mobile health technologies to justify that any investments in hardware or information technology connections are offset by lower health care expenditures and better outcomes. For example, it will be helpful to demonstrate that more personalized care leads to improved health of patients and reductions in the number of planned or unplanned hospital admissions. Such research should also provide a benchmark for discussion between payers and commercial companies, ascertaining reasonable pricing that will help with its implementation and long-term sustainability.

\section{Anticipated Challenges}

An obvious and enormous challenge ahead is the change in behavior required of both patients and professionals to ensure widespread adoption and long-term use and of regulators and health care systems to maintain 
such platforms. The financial model must be sustainable and the full extent of regulatory hurdles fully understood and overcome. To this end, the ownership of running, managing, and administrating open-source platforms would be ideal for an organization such as MDS to take on. A pilot use of competing platforms might be considered as an exploratory step to inform the one providing the best clinical value and integration with developers as well as technology and health care industries. The final major challenge is that of seamlessly coupling the outcome of mobile health technologies to the existing information technology infrastructure at hospitals such as in electronic medical records as well as determining the financial costs associated with using and maintaining (updating) periodic technological improvements.

\section{Conclusions and Next Steps}

The time has come to further develop and integrate mobile health technologies into the routine assessment and care of patients with PD. The improvements in the sophistication, versatility, and wearability of these technologies have reached a state of maturity that is adequate for the collection of patient-relevant data. To harness these opportunities, the MDS Task Force on Technology recommends that mobile health technologies will need to (1) target deficits confirmed to be relevant to patients; 2 ) be derived from ideally a single or else a combination of devices that deliver an acceptable benefit-to-burden ratio to patients and at the same time yield clinically useful information; (3) be integrated and synchronized into patient management platform standards, delivering individualized data to patients, caregivers, and clinicians; and (4) be approved by regulators, weaved into digital health systems, and uniformly adopted by health care delivery organizations. Added value and appropriate cost-benefit ratios still need to be determined before MDS or any other PD-focused organization in its place can assume the ownership of hiring expert technology services to run a platform and coordinate its maintenance and administration (including dynamic decisionmaking on which elements to include or exclude). This collaborative endeavor will encourage the development of integrated, multichannel systems that can achieve more sophisticated characterization of patients' function, better tailoring of symptomatic therapy, greater patient engagement and self-assessment by patients, and overall improved health care outcomes.

\section{References}

1. Espay AJ, Bonato P, Nahab FB, et al. Technology in Parkinson's disease: challenges and opportunities. Mov Disord 2016;31(9):1272-1282.
2. Godinho C, Domingos J, Cunha G, et al. A systematic review of the characteristics and validity of monitoring technologies to assess Parkinson's disease. J Neuroeng Rehabil 2016;13:24.

3. van Uem JM, Isaacs $\mathrm{T}$, Lewin $\mathrm{A}$, et al. A viewpoint on wearable technology-enabled measurement of wellbeing and health-related quality of life in Parkinson's disease. J Parkinsons Dis 2016;6(2):279-287.

4. Artusi CA, Mishra M, Latimer P, et al. Integration of technologybased outcome measures in clinical trials of Parkinson and other neurodegenerative diseases. Parkinsonism Relat Disord 2018;46 (suppl 1):S53-S56.

5. Merola A, Sturchio A, Hacker S, et al. Technology-based assessment of motor and nonmotor phenomena in Parkinson disease. Expert Rev Neurother 2018;18(11):825-845.

6. Lord S, Godfrey A, Galna B, Mhiripiri D, Burn D, Rochester L. Ambulatory activity in incident Parkinson's: more than meets the eye? J Neurol 2013;260(12):2964-2972.

7. Mandl KD, Kohane IS. A 21st-century health IT System-creating a real-world information economy. N Engl J Med 2017;376(20): 1905-1907.

8. Mandl KD, Kohane IS. Time for a patient-driven health information economy? N Engl J Med 2016;374(3):205-208.

9. Merchant KM, Cedarbaum JM, Brundin P, et al. A proposed roadmap for parkinson's disease proof of concept clinical trials investigating compounds targeting alpha-synuclein. J Parkinsons Dis 2019;9(1):31-61.

10. Mirelman A, Gurevich T, Giladi N, Bar-Shira A, Orr-Urtreger A, Hausdorff JM. Gait alterations in healthy carriers of the LRRK2 G2019S mutation. Ann Neurol 2011;69(1):193-197.

11. Berg D, Postuma RB, Adler CH, et al. MDS research criteria for prodromal Parkinson's disease. Mov Disord 2015;30(12):1600-1611.

12. Lipsmeier F, Taylor KI, Kilchenmann T, et al. Evaluation of smartphone-based testing to generate exploratory outcome measures in a phase 1 Parkinson's disease clinical trial. Mov Disord 2018;33 (8):1287-1297.

13. Heldman DA, Espay AJ, LeWitt PA, Giuffrida JP. Clinician versus machine: reliability and responsiveness of motor endpoints in Parkinson's disease. Parkinsonism Relat Disord 2014;20(6): 590-595.

14. Silva de Lima AL, Hahn T, Evers LJW, et al. Feasibility of largescale deployment of multiple wearable sensors in Parkinson's disease. PLoS One 2017;12(12):e0189161.

15. De Fauw J, Ledsam JR, Romera-Paredes B, et al. Clinically applicable deep learning for diagnosis and referral in retinal disease. Nat Med 2018;24(9):1342-1350.

16. Zhan A, Mohan S, Tarolli C, et al. Using smartphones and machine learning to quantify Parkinson disease severity: the Mobile Parkinson Disease Score. JAMA Neurol 2018;75(7):876-880.

17. Frohlich H, Balling R, Beerenwinkel N, et al. From hype to reality: data science enabling personalized medicine. BMC Med 2018;16(1):150.

18. World Health Organization. International Classification of Functioning, Disability and Health. Geneva, Switzerland: World Health Organization; 2001.

19. Bravell ME, Zarit SH, Johansson B. Self-reported activities of daily living and performance-based functional ability: a study of congruence among the oldest old. Eur J Ageing 2011;8(3):199-209.

20. Arbatti L, Marras C, Standaert DG, Tanner CM, Nguyen A, Shoulson I. Problems that bother Parkinson patients: the basis for a patient-reported natural history. Ann Neurol 2018;84:S1-S280.

21. Serrano JA, Larsen F, Isaacs T, et al. Participatory design in Parkinson's research with focus on the symptomatic domains to be measured. J Parkinsons Dis 2015;5(1):187-196. 